Lateral capillary forces of cylindrical fluid menisci: a comprehensive quasi-static study

This article has been downloaded from IOPscience. Please scroll down to see the full text article.

2010 J. Micromech. Microeng. 20075041

(http://iopscience.iop.org/0960-1317/20/7/075041)

View the table of contents for this issue, or go to the journal homepage for more

Download details:

IP Address: 128.178.244.221

The article was downloaded on 09/11/2010 at 17:38

Please note that terms and conditions apply. 


\title{
Lateral capillary forces of cylindrical fluid menisci: a comprehensive quasi-static study
}

\author{
M Mastrangeli ${ }^{1,2}$, J-B Valsamis ${ }^{3}$, C Van Hoof ${ }^{1,4}$, J-P Celis ${ }^{2}$ and \\ P Lambert ${ }^{3,5}$ \\ ${ }^{1}$ IMEC, Kapeldreef 75, B-3001 Leuven, Belgium \\ ${ }^{2}$ MTM Department, Katholieke Universiteit Leuven, Kasteelpark Arenberg 44, B-3001 Leuven, Belgium \\ ${ }^{3}$ BEAMS Department, Université Libre de Bruxelles, Avenue F. D. Roosevelt CP 165/56 50, B-1050 \\ Bruxelles, Belgium \\ ${ }^{4}$ ESAT Department, Katholieke Universiteit Leuven, Kasteelpark Arenberg 10, B-3001 Leuven, Belgium \\ ${ }^{5}$ Femto-ST Institute, CNRS UMR 6174, 24 Rue Alain Savary, F-25000 Becançon, France \\ E-mail: mastrangelim@gmail.com
}

Received 18 February 2010, in final form 30 April 2010

Published 28 June 2010

Online at stacks.iop.org/JMM/20/075041

\begin{abstract}
Capillarity is pivotal to many important technologies, including capillary self-alignment and self-assembly for heterogeneous microsystem integration and packaging. Lateral capillary forces ensuing from perturbed fluid menisci were the object of substantial theoretical and numerical modeling in recent years. Anyway, those studies were so far unsatisfactorily supported by direct experimental inspections. In this paper we present a comprehensive quasi-static study of lateral capillary forces arising from a constrained cylindrical fluid meniscus subjected to small lateral perturbations. We describe the novel experimental apparatus that we designed to accurately characterize such a fundamental system. We then reproduce our experimental data on lateral meniscus forces and stiffnesses by means of both a finite element and a novel analytical model. The agreement between our measurements and models, while confirming earlier reports, provides a solid foundation for the applications of lateral capillary forces to microsystem assembly. Moreover, our experimental apparatus may enable the exploitation of Gibbs' inequality to measure the advancing contact angles of liquids, and it may be used as a reference testbed for further experimental investigations on constrained fluid menisci.
\end{abstract}

(Some figures in this article are in colour only in the electronic version)

\section{Introduction}

Capillary and surface-tension-related phenomena are ubiquitous in everyday life. Their thermodynamic origin lies in the minimization of the free energy of physical systems containing non-rigid interfaces $[1,2]$. Fundamental in biochemistry [3], capillary phenomena are presently at the core of many important technologies as well, such as e.g. switchable lenses [4], tightness systems [5], precision manufacturing [6], metal bonding and soldering [7], microfabrication and microfluidics [8], and integration and packaging of heterogeneous microsystems [9].
With specific focus on the latter, the molten solderdriven self-aligning motion of IC dies on top of pre-processed substrates was first exploited in flip-chip assembly to achieve highly accurate passive die registration [10-13]. Capillarity is one powerful enabler of the three-dimensional deployment and actuation of articulated microelectromechanical structures of unprecedented complexity [14, 15] and it is one of the physical mechanisms driving the growing class of packaging techniques based on self-assembly [16]. Surface-tensiondriven aggregation was first adopted for the self-assembly of heterogeneous functional systems by Whitesides' group [17-19]. It was later adapted to part-to-substrate assembly 
tasks by the teams of Howe [20, 21], Böhringer [22], Koyanagi [23, 24], Parviz [25, 26] and Jacobs [27, 28], to mention but a few examples.

Most aforementioned assembly techniques share, at least partly, the same underlying mechanism. Upon contact between the functional (i.e. suitably pre-conditioned to enable further processing) side of the part to be assembled (hereby representing e.g. an IC die, a microdevice, a MEMS component) and the highly energetic mating surfacecomposed of a fluid, such as e.g. hydrocarbons [20], water-based solutions [23] or molten solders [29]—of the corresponding binding site on the substrate, capillary torques and forces - both perpendicular (i.e. vertical) and parallel (i.e. lateral) to the substrate-act on the part and drive it to selfalign with the underlying binding site [30]. Floating on the liquid meniscus and aligning to the pattern of the binding site, the part achieves its rest (i.e. minimal energy) configuration. The same capillary forces intervene to oppose any sufficiently small displacement of the part from its rest position - they are therefore also referred to as restoring forces. The accuracy of registration between part and binding site critically depends on lateral capillary forces - the object of the present contribution. Capillary torque and vertical capillary meniscus forces were investigated, among others, by Takei et al [31] and by Lambert [6], respectively.

Early theoretical investigations on lateral capillary forces addressed the shapes and corresponding self-aligning performance of fluid drops (representing e.g. molten-solder bumps) vertically constrained by parallel plates and laterally confined by the geometrical patterns, of contrasting wetting properties, defined on the same plates (i.e. the binding sites) [32-34]. Finite-element quasi-static numerical simulations of a similar physical system-where the top plate was represented by a part of finite dimensions and free in all translational and rotational degrees of freedom (DOFs) - further illustrated the dependence of lateral capillary forces on the physical properties of the fluid meniscus and on the geometry of the confining geometrical patterns [35, 36]. Briefly, the forces are proportional to the surface tension of the fluid and inversely proportional to the height of the meniscus (also referred to as the gap in the following), and they follow the (a)symmetry of the patterns of the confining sites: equal relative displacements along shorter sides of the sites originate stronger forces. Böhringer proposed a geometrical model of confined capillary menisci that greatly simplified energy and force calculations [37]. Built around the twodimensional convolution of the patterns of the confining surfaces, the model eased the theoretical investigations on the geometry-dependent self-alignment performance of binding sites $[38,39]$. Nonetheless, being essentially twodimensional, Böhringer's model accurately reproduces the known results only for relative displacements from the rest position larger than the meniscus height. Thus it is not suitable to model the alignment performance in close proximity to the rest position-which is conversely of utmost importance for our interest in flip-chip-like assembly of dies with very small interconnection pitches (e.g. few tens of microns). Particularly, the model neglects the curvature of the surface of the fluid meniscus, which affects the capillary restoring forces particularly for small meniscus perturbations [35, 40]. Given the fixed geometrical boundary conditions, the meniscus curvature is directly influenced by the volume of the fluid: hence the need for accurate fluid volume control to achieve high process reliability and reproducibility [41].

As compared to the extensive modeling literature, experimental quasi-static investigations of lateral capillary forces were up to now overall unsatisfactory. A few works reported on the determination of the ultimate registration accuracy achievable between part and binding site by means of capillary self-alignment: measurements were performed either optically $[20,42,43]$ or by analyzing assembly crosssections by scanning electron microscopy [24]. Best claimed figures were of the order of $1 \mu \mathrm{m}$ or lower-amenable to advanced packaging applications. Other researchers [44, 45] performed blowing tests [46] to estimate the maximum adhesion force binding flat parts onto confined fluid drops. However, under such conditions-where the direction of the displacement of the floating part was not constrained to be parallel to the substrate-the estimated force values may hardly be attributed only to lateral force components. To our knowledge, only Zhang et al reported experimental measurements and numerical simulations of lateral capillary forces for the same physical system [47]. The system, immersed in water, was composed by a $450 \mu \mathrm{m} \times 250 \mu \mathrm{m}$ flat thin silicon piece floating on a matching rectangular site confining a drop of hydrophobic fluid. Lateral capillary forces were measured in situ by means of a micromachined optical encoder featuring laser-illuminated, calibrated optical gratings and a horizontal probe that displaced the part from its rest position along a direction parallel to the substrate. Anyway, though the proposed numerical model showed a reasonable match with experimental data, no mention was thereby to be found of e.g. the surface tension and volume of the fluid and of the meniscus height-pivotal parameters to reproduce and appreciate the numerical results. Moreover, the way the physical system was set up, though consistent with their proposed application, was not easily controllable, and only one experimental measurement was shown.

In this paper we present a comprehensive quasistatic study of lateral capillary meniscus forces, in which experimental, numerical and analytical analyses performed on the same system are compared. We describe the conceptually simple experimental setup that we designed to quasi-statically measure the lateral restoring forces arising from small perturbation of fluid menisci confined between two parallel plates (section 2). Easy to use and macroscopic, our setup still allowed us to control the position of the movable plates with $1 \mu \mathrm{m}$ accuracy, and to resolve forces as low as about $1 \mu \mathrm{N}$. By fixing the dimensions of the confining pads and the meniscus fluid and height beforehand, the setup allowed for repeatable experiments. Our setup may be roughly likened to an upscaled version of the flexure-based microgap rheometer developed by Clasen et al to study microgap-dependent flow behavior of complex fluids [48]. The experimental results obtained for circular pads were then supported by both a finite-element (section 3) and an analytical model (section 4) 


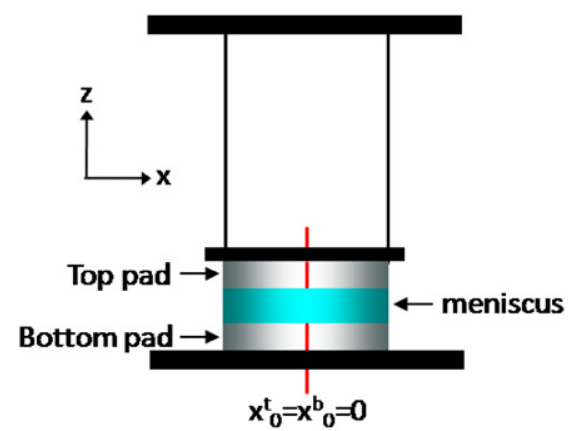

(a)

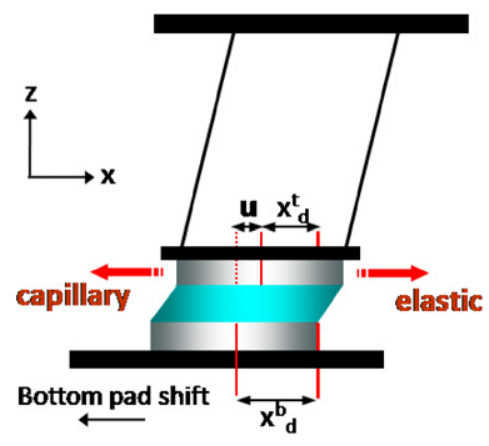

(b)

Figure 1. The sensing principle, based on the balance between capillary and elastic lateral forces. Relative dimensions are out of scale for illustration purposes. (Actual examples of rest and perturbed configurations are shown in figure 2.) (a) Rest configuration. $(b)$ Perturbed configuration.

predicting restoring forces for small perturbation of cylindrical fluid menisci, with good accuracy (section 5). Given the proper boundary conditions, our sensing apparatus may also incidentally enable a novel, overflow method to measure advancing contact angles of liquids. In view of our results, our setup may constitute a reference testbed for further investigations on confined fluid menisci (section 6).

Finally, while the present contribution focuses on statics only, the dynamics of capillary self-alignment is investigated in a companion paper [49].

\section{Experimental measurement of lateral capillary meniscus forces}

\subsection{Requirements and constraints}

In designing our sensing apparatus, we tried to satisfy several requirements at once:

(i) we required a simple apparatus for precise spatial manipulations;

(ii) the apparatus had to enable carefully controlled and repeatable experiments: all relevant meniscus parameters of each experiment had to be reliably known (before or after the experiment itself);

(iii) we were interested in precisely measuring lateral capillary forces of the same order of magnitude as those arising in flip-chip-like assembly, i.e. forces reportedly of the order of tens of $\mu \mathrm{N}$ [36];

(iv) we required to measure only lateral capillary forces;

(v) we aimed at sampling the capillary force-versus-lateral displacement curve close to its origin, i.e. for lateral displacements smaller that the meniscus height;

(vi) finally, the fluids to be used had to have low volatility, so to allow up to tens of minutes long quasi-static measurements.

\subsection{The sensing principle}

Our experimental setup was built around a fluid meniscus confined between two horizontal parallel circular pads and monitored by a mechanical spring (as detailed in section 2.3).

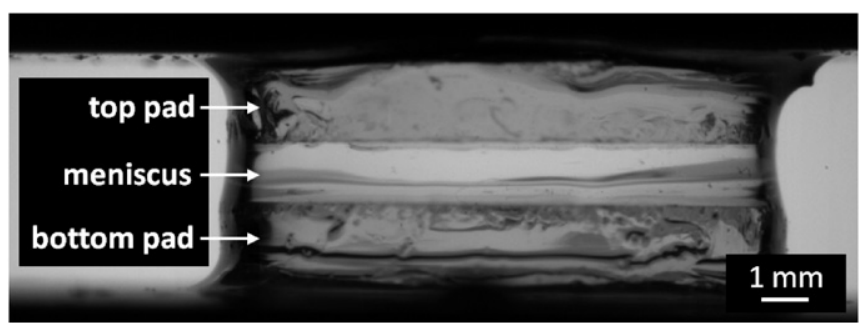

$(a)$

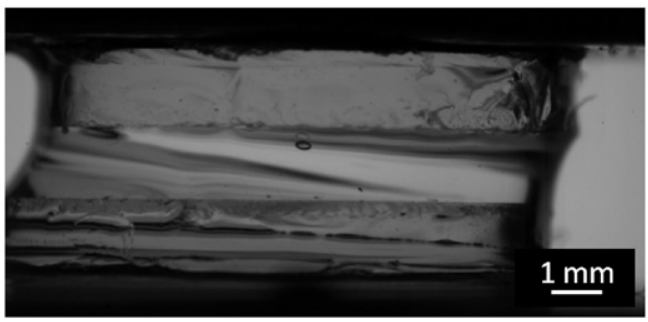

(b)

Figure 2. Measurement of lateral capillary forces of a cylindrical meniscus. (a) Rest configuration. Top and bottom pads are aligned, and the height of the meniscus $(1.205 \mu \mathrm{m})$ is trimmed to get a cylindrical profile. (b) Perturbed configuration. After imposing a displacement $\left(x_{d}^{b}=812 \mu \mathrm{m}\right)$ on the bottom pad, the lateral force balance between capillary and elastic spring forces determines the equilibrium displacement $(u=660 \mu \mathrm{m})$ of the top relative to the bottom pad.

The setup embodied a sensing principle based on the balance between capillary and elastic lateral forces (figure 1).

As for initial conditions, the vertical superposition and distance of the centers of bottom and top pads (with coordinates $x^{b}$ and $x^{t}$, respectively) were set so as to enforce a cylindrical meniscus profile (figure $2(a)$ ). In this state, both the fluid meniscus and the spring were in their rest configurations, defined by their reference starting positions $x_{0}^{b}=x_{0}^{t}=0$ (figure 1(a)).

The measurements were performed by imposing a known lateral displacement $x_{d}^{b}$ on the bottom pad (figures $1(b)$ and 2(b)). As a consequence of this lateral perturbation, two opposing lateral forces acted on the top pad: the capillary force tends to restore the meniscus rest position, and the elastic spring force tends to restore the spring rest position. The equilibrium of the lateral forces consequently determined the 


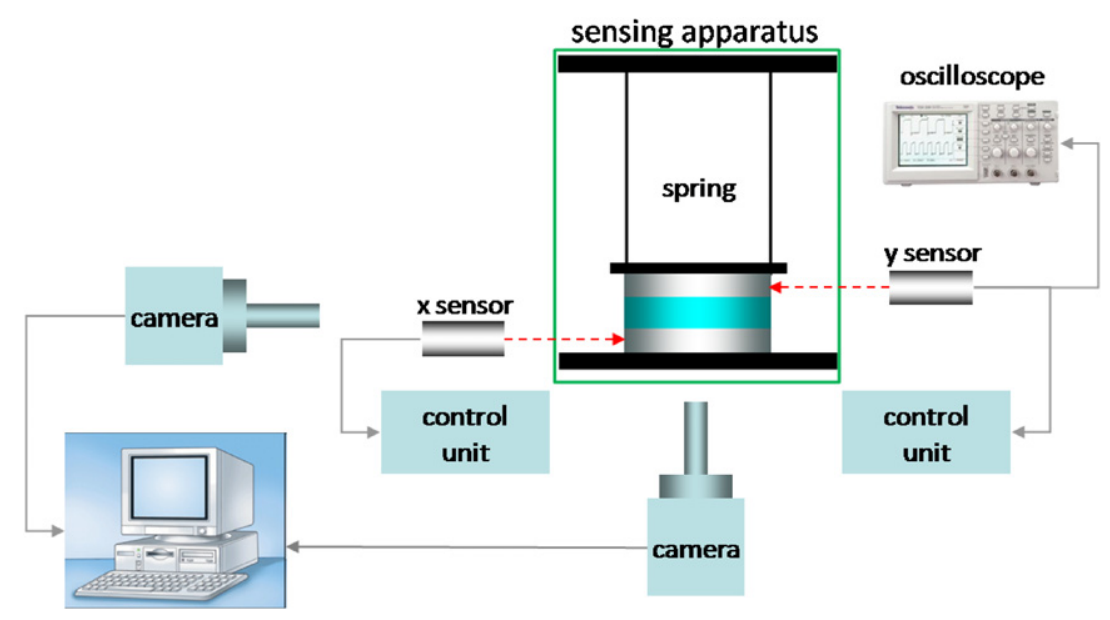

Figure 3. Schematic representation of the experimental setup. Relative dimensions are out of scale for representation purposes.

actual displacement of the top pad: this was $x_{d}^{t}$ with respect to the rest position of the spring, and $u=x_{d}^{b}-x_{d}^{t}$ relative to the bottom pad. Hence, the restoring lateral meniscus force corresponding to the net displacement $u$ of the top pad relative to the bottom pad could be calculated by multiplying the absolute displacement $x_{d}^{t}$ of the top pad times the bending stiffness of the spring $K$-whose estimation is described in appendix A.

By imposing series of temporally isolated displacements to the bottom pad, we could experimentally measure the lateral restoring force as a function of the relative lateral displacement of our physical system. The lateral stiffness of each fluid meniscus $K_{m}^{L}$ was obtained by the first-order polynomial fitting of this curve. In all experiments the same set $\left\{x_{d_{i}}^{b}\right\}$ of bottom pad displacements was imposed in both directions (i.e. forward and backward scans) around the initial position, in order to ascertain eventual asymmetries and/or hysteretic phenomena.

\subsection{The experimental setup}

The schematic diagram and the actual experimental setup are shown in figures 3 and 4, respectively. Two equal glass pads (diameter $D=2 \cdot R=9.4 \mathrm{~mm}$, thickness $=1.6 \mathrm{~mm}$ ) confined and shaped the liquid menisci under test. The bottom pad was solidal to an anchored 6-DOFs stage (Newport M-562-XYZ) that could be manually roto-translated with micrometric resolution, as tracked by a laser displacement sensor (Keyence LC-2440) working within its linear output range. The sensor's controller unit (Keyence LC-2400W) displayed real-time-averaged displacements. The top pad was solidal to the bottom surface of a finely machined aluminum parallelepiped (the shuttle of mass $m_{\mathrm{sh}}=12.748 \mathrm{~g}$ including the top pad), in turn held by two equal steel cantilevers (Precision Brand) of certified dimensions and total mass $m_{b}=2 \cdot 2.85 \mathrm{~g}$ hanging from an overarching solid bridge (not shown). This double-cantilever-supported shuttle constituted the actual sensing apparatus, i.e. the spring. The sliding motion of the shuttle (i.e. the bending motion of the cantilevers) was thus constrained to take place exclusively along the chosen lateral measurement direction (see also footnote 7), thereby tracked by an independent laser displacement sensor (and control unit). The hanging point and the length of the spring cantilevers (and so the vertical position of the top pad) were never modified after initial calibration (described below): we used exactly the same apparatus in all experiments. Accordingly, (1) the height $h$ of the fluid menisci was set using only the controlled vertical motion of the translation stage; (2) the vertical components of the capillary force were excluded from measurements (requirement (iv)), being balanced by the vertical vincular reactions of the rigid supports.

To check the pad alignment, plane-parallelism of pad surfaces and fluid meniscus profile, top and bottom pads were visually tracked by two PC-connected cameras: a USB camera pointed along the measurement direction, and a highspeed camera (Photron Fastcam SA3 120K) pointed along a perpendicular direction intersecting the former at the initial position of the bottom pad. The pads were illuminated by a flat backlight (LDL-TP-83×75, with CCS PD-3012 power supply unit) with uniform red light emission profile and unnoticeable heating power. For every experiment, pictures of the menisci were taken with the high-speed camera right after the initial meniscus trimming and at the end of measurements. The 1024 pixel wide pictures spanned a field of view of about $20 \mathrm{~mm}$, providing a positioning accuracy of less than $20 \mu \mathrm{m}$.

\subsection{Fluids}

A series of homologous silicone oils were used as reference fluids (table 1). All the experiments were performed in a laboratory environment: room temperature varied between $25^{\circ} \mathrm{C}$ and $28^{\circ} \mathrm{C}$; the monitored relative air humidity was about $38 \%$.

The capillary length $L_{c}=\sqrt{\frac{\gamma}{\rho g}}$ of the oils was about $1.49 \mathrm{~mm}$. The imposed heights of the menisci were smaller than $L_{c}$ in all experiments, so gravity could be neglected as compared to capillary forces (requirement (iv)). For each fluid, we performed a series of experiments with progressively smaller meniscus heights. In the first experiment of each series, the meniscus height was calibrated to exactly $1 \mathrm{~mm}$ by means of a ceramic slip gage of certified thickness (Mitutoyo). The contact angle (CA or $\theta$ in the following) of silicone 


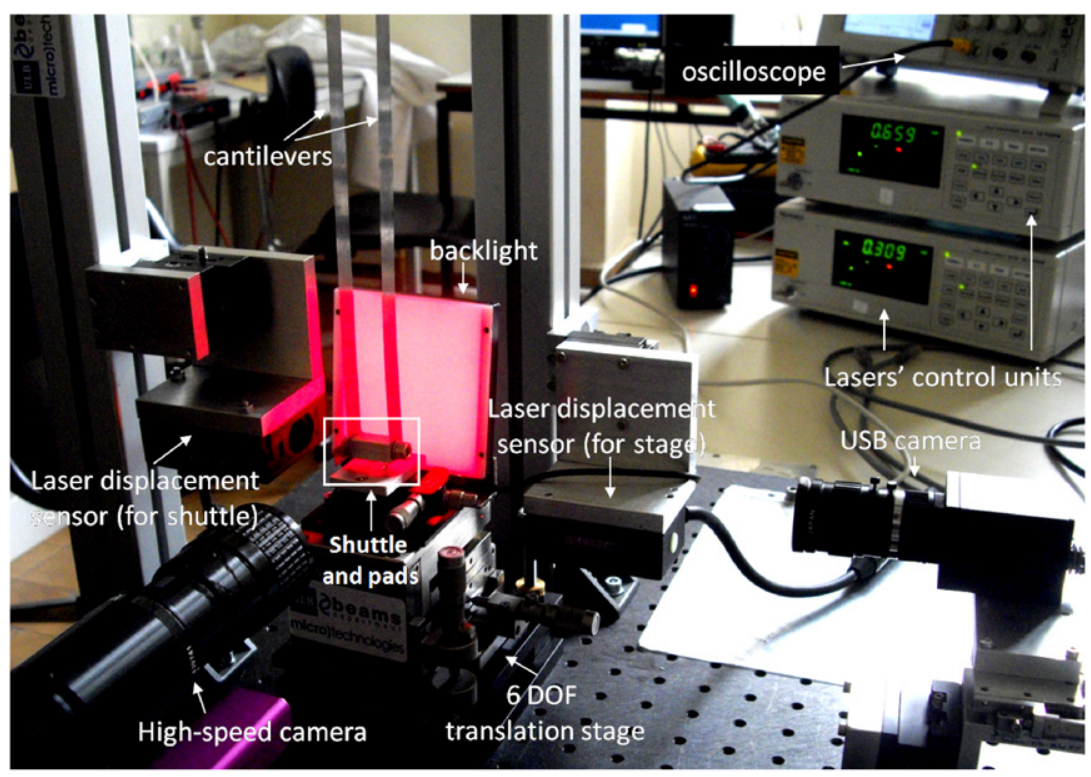

(a)

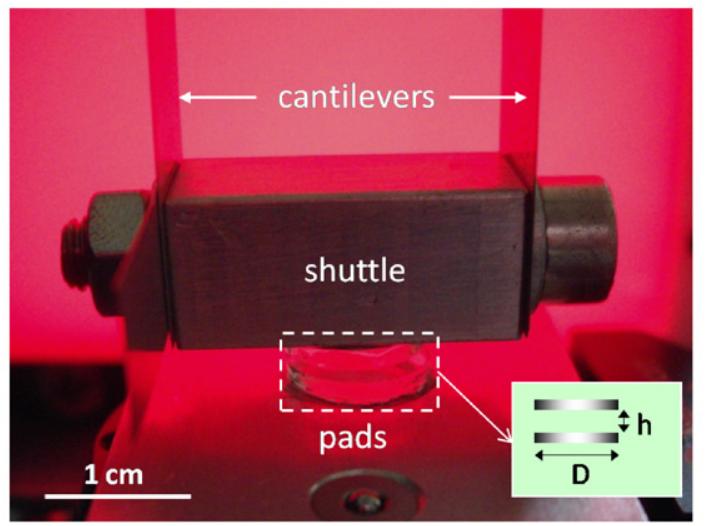

(b)

Figure 4. The experimental setup for lateral capillary force measurements. (a) Full view. (b) Close-up view of the inset in $(a)$.

Table 1. Fluids used in the experiments.

\begin{tabular}{lccl}
\hline & $\begin{array}{l}\text { Surface } \\
\text { Name } \\
\left(\mathrm{kg} \mathrm{m}^{-3}\right)\end{array}$ & $\begin{array}{l}\text { tension } \\
\left(\mathrm{N} \mathrm{m}^{-1}\right)\end{array}$ & Supplier \\
\hline Oil 1 & 970 & 0.0211 & Rhodorsil R47V500 \\
Oil 2 & 973 & 0.0211 & Rhodorsil R47V5000 \\
Oil 5 & 934 & 0.0201 & Dow Corning DC200FLUID10 \\
Oil 6 & 960 & 0.0209 & Dow Corning DC200FLUID100 \\
Oil 7 & 971 & 0.0212 & Dow Corning DC200FLUID1000 \\
Water & 1000 & 0.072 & Tap water \\
\hline
\end{tabular}

oils on clean hydrophilic surfaces is reportedly rather small [1]. This, combined with the geometric constraint captured by Gibbs' inequality [50] (discussed in section 5.2), sets an upper bound on the maximum allowed perturbation of the fluid meniscus to avoid fluid overflow beyond the edges of the bottom pad (and a consequent change in the volume of the fluid meniscus). Nevertheless, the range of relative pad displacements still allowed was fully coherent with our focus on small lateral meniscus perturbations (i.e. requirement (v)). Effects of oil evaporation were negligible throughout all our experiments, consistent with their low volatility. In contrast, in some explorative experiments we also used water as meniscus fluid $\left(\gamma=72 \mathrm{~mJ} \mathrm{~m}^{-2}, L_{c}^{\mathrm{H}_{2} \mathrm{O}}=2.72 \mathrm{~mm}\right)$; as expected, its high volatility made our quasi-static measurements problematic and difficult to reproduce (see section 5.2).

\subsection{Data processing}

The fluids were dispensed between the pads by manual pipetting from calibrated pipettes. Because of the intrinsic dispensing imprecision due to hardly controllable tip pinch-off effects, we calculated the actual volumes $V$ and height $h$ of the fluid menisci by digital post-processing of the pictures taken at the beginning of the experiments. The exact calibration of the images, done using the $1 \mathrm{~mm}$ thick ceramic gage, led to a spatial resolution of $14.2 \mu \mathrm{m} /$ pixel. Our semi-automatic numerical algorithm computed $V, h$ and $D$ for each experiment assuming axial symmetry of the menisci. We set the meniscus axisymmetry experimentally: for each dispensed fluid drop, we set an initial cylindrical meniscus profile (i.e. a strictly vertical lateral meniscus surface, as assumed in the analytical model, see section 4), as judged by visual camera inspection 


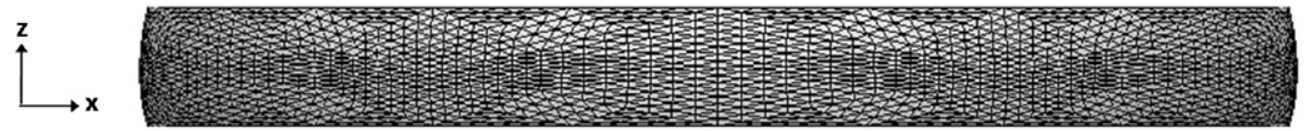

(a)

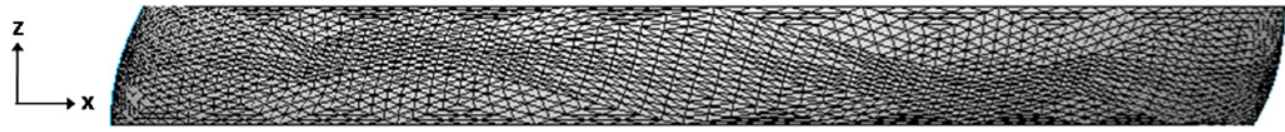

(b)

Figure 5. The finite-element model of the fluid meniscus defined in experiment 2 of table 2. (a) Rest configuration. (b) Maximally perturbed configuration $(u=273 \mu \mathrm{m})$.

Table 2. Summary of results (Exp = experimental, $\mathrm{SE}=$ surface evolver, $\mathrm{An}=$ analytical). The relative errors of the SE and An models compared to the experimental estimates are shown in the last column. The error (relative to the measured value; indicated by RE in the second column) in the reconstruction of the pad diameter by the image post-processing algorithm was used to assess the accuracy of the estimates on meniscus heights and volumes.

\begin{tabular}{|c|c|c|c|c|}
\hline Experiment & Geometry & Fluid & $K\left(\mathrm{~N} \mathrm{~m}^{-1}\right)$ & Modeling error \\
\hline 1 & $\begin{array}{l}\text { Height: } 1.138 \mathrm{~mm} \\
\text { Volume: } 85.6 \mathrm{~nL} \\
\text { Diameter: } 9.186 \mathrm{~mm} \text { (RE: } 2.28 \% \text { ) }\end{array}$ & Oil 2 & $\begin{array}{l}\text { Exp: } 0.2466 \\
\text { SE: } 0.2415 \\
\text { An: } 0.2797\end{array}$ & $\begin{array}{r}2.07 \% \\
13.42 \%\end{array}$ \\
\hline 2 & $\begin{array}{l}\text { Height: } 1 \mathrm{~mm} \\
\text { Volume: } 74.2 \mathrm{~nL} \\
\text { Diameter: } 9.63 \mathrm{~mm} \text { (RE: } 2.45 \% \text { ) }\end{array}$ & Oil 6 & $\begin{array}{l}\text { Exp: } 0.2827 \\
\text { SE: } 0.2940 \\
\text { An: } 0.3152\end{array}$ & $\begin{array}{r}3.99 \% \\
11.48 \%\end{array}$ \\
\hline 3 & $\begin{array}{l}\text { Height: } 0.852 \mathrm{~mm} \\
\text { Volume: } 57.1 \mathrm{~nL} \\
\text { Diameter: } 9.41 \mathrm{~mm} \text { (RE: } 0.11 \% \text { ) }\end{array}$ & Oil 6 & $\begin{array}{l}\text { Exp: } 0.3392 \\
\text { SE: } 0.3456 \\
\text { An: } 0.3698\end{array}$ & $\begin{array}{l}1.89 \% \\
9.01 \%\end{array}$ \\
\hline 4 & $\begin{array}{l}\text { Height: } 1 \mathrm{~mm} \\
\text { Volume: } 65.5 \mathrm{~nL} \\
\text { Diameter: } 9.29 \mathrm{~mm} \text { (RE: } 1.21 \%)\end{array}$ & Oil 2 & $\begin{array}{l}\text { Exp: } 0.2407 \\
\text { SE: } 0.2648 \\
\text { An: } 0.3182\end{array}$ & $\begin{array}{l}10.01 \% \\
32.19 \%\end{array}$ \\
\hline 5 & $\begin{array}{l}\text { Height: } 0.906 \mathrm{~mm} \\
\text { Volume: } 54.2 \mathrm{~nL} \\
\text { Diameter: } 9.15 \mathrm{~mm} \text { (RE: } 2.64 \% \text { ) }\end{array}$ & Oil 2 & $\begin{array}{l}\text { Exp: } 0.2962 \\
\text { SE: } 0.3285 \\
\text { An: } 0.3510\end{array}$ & $\begin{array}{l}10.90 \% \\
18.50 \%\end{array}$ \\
\hline 6 & $\begin{array}{l}\text { Height: } 0.720 \mathrm{~mm} \\
\text { Volume: } 47.3 \mathrm{~nL} \\
\text { Diameter: } 9.44 \mathrm{~mm} \text { (RE: } 0.42 \%)\end{array}$ & Oil 2 & $\begin{array}{l}\text { Exp: } 0.3485 \\
\text { SE: } 0.3989 \\
\text { An: } 0.4417\end{array}$ & $\begin{array}{l}14.46 \% \\
26.75 \%\end{array}$ \\
\hline
\end{tabular}

(see e.g. figure 2(a)). We made three numerical estimates on each calibration picture, and we a posteriori discarded all experiments for which the ratio between the average values of $V$ and $h$ and their standard deviation was larger than 10 . The uncertainty in the heights was typically 2 pixels, i.e. about $28 \mu \mathrm{m}$ : thus we kept the heights of the menisci always higher than $280 \mu \mathrm{m}$. Moreover, by comparing the reconstructed value of $D$ to its caliber-measured value, we could assess the accuracy of the numerical estimates of $D, V$ and $h$ (see table 2).

We remark that, though we used only circular pads in our experiments, fluid menisci shaped by pads of arbitrary geometries can be investigated with our setup - though exact knowledge of the dispensed fluid volume may be harder to obtain for non-axisymmetric menisci.

\section{Finite-element model}

We modeled our experiments on cylindrical fluid menisci with Surface Evolver (SE) [51] (figure 5). SE is powerful finite- element software dedicated to the computation of energyminimizing surfaces defined by geometrical and physical constraints [52]. Its mathematical solver works essentially in $2 \mathrm{D}$, i.e. the energies to be minimized are quantities that may be defined as integrals over surfaces. This is straightforward for the surface tension of the triangular facets of the body. To account for the gravitational potential energy $E_{g}$ of a body of volume $\Omega$, surface $\partial \Omega$ and uniform density $\rho$, the required surface integral (computed for each facet bounding the body) is derived from the divergence theorem as follows [53]:

$$
E_{g}=g \rho \iiint_{\Omega} z \mathrm{~d} V \underset{\text { divergence }}{=} g \rho \iint_{\partial \Omega} \frac{z^{2}}{2} \vec{k} \cdot \mathrm{d} \vec{S}
$$

where $\vec{k}$ and $\mathrm{d} \vec{S}$ are the unit vectors parallel to the vertical direction of the local frame of reference and normal to the facet surfaces, respectively.

Our code was adapted from the column example written by SE's developer Brakke ${ }^{6}$. All geometrical (radius of the pads

6 The original column code is available at
http://www.susqu.edu/brakke/evolver/html/column.htm 


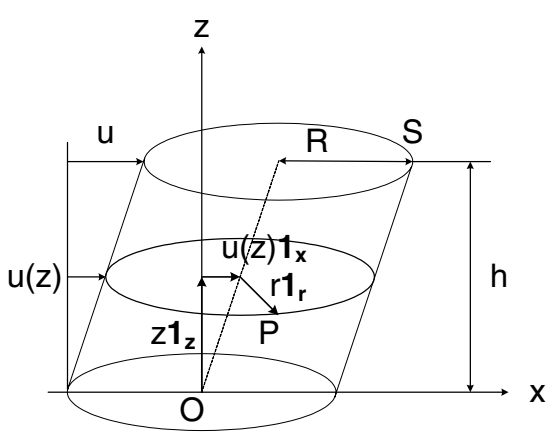

Figure 6. Geometrical model of a fluid meniscus of height $h$ confined between circular parallel pads of radius $R$ laterally offset by $u$.

$R$, meniscus height and volume as obtained by image postprocessing for each experiment) and physical (fluid density and surface tension) parameters reproduced those of the experiments chosen to be modeled. We imposed the pinning of the fluid triple contact-line along all the circular edges of both pads. This condition was enforced at the beginning of each experiment, as already said, and was satisfied for all measurements of small meniscus perturbations, as judged by visual inspection. The angle between the lateral meniscus surface and the pad surfaces at the triple contact-line was therefore not imposed, rather a consequence of the physical (i.e. fluid surface tension) and geometrical (fluid volume and pad areas) boundary conditions of the problem.

The capillary forces were calculated by the method of virtual works (see e.g. [54], p 25) using central differences. Our script for mesh refinement and geometry evolution made repeated use of the Hessian of the energy function, achieving energy convergence to its fifth significant digit and a satisfactory match $(<1 \%)$ against the benchmark given by the axial force produced by a perfectly cylindrical fluid meniscus-analytically given by $F=-\pi \gamma R$ [6]. In simulating the force-versus-displacement curve of a few of our experiments, we input the same set $\left\{u_{i}\right\}$ of relative displacements that were observed in the experiments. We finally extrapolated the simulated lateral stiffness of the fluid menisci by the first-order polynomial fitting of the curves.

\section{Analytical model}

We developed an analytical model to estimate the lateral stiffness of a cylindrical fluid meniscus confined between two circular pads (figure 6). Perfectly cylindrical meniscus profiles were assumed for analytically closed-form tractability, though such assumption is admittedly ideal and not always satisfied under experimental conditions.

We firstly computed the lateral area of a tilted cylinder of height $h$, radius $R$ and relative lateral shift between pads $u<h$. In this configuration, the cylinder axis is not perpendicular to both pads but inclined with an angle $\alpha$ given by $\tan \alpha=u / h$.

The equation of this cylinder is given by

$$
\begin{aligned}
S & \equiv \overline{O P}=z \overline{1}_{z}+u(z) \overline{1}_{x}+R \overline{1}_{r} \\
& =\left(\frac{z}{h} u+R \cos \theta\right) \overline{1}_{x}+(R \sin \theta) \overline{1}_{y}+z \overline{1}_{z} .
\end{aligned}
$$

Computing the area element $\mathrm{d} S$ as

$$
\mathrm{d} S=\|\bar{N}\| \mathrm{d} \theta \mathrm{d} z
$$

with $\bar{N}=\frac{\partial \bar{S}}{\partial \theta} \times \frac{\partial \bar{S}}{\partial z}$, we finally find

$$
\mathrm{d} S=R \sqrt{1+\cos ^{2} \theta \frac{u^{2}}{h^{2}}} \mathrm{~d} \theta \mathrm{d} z .
$$

The lateral area is consequently equal to

$$
S=R \int_{0}^{h} \mathrm{~d} z \int_{0}^{2 \pi} \sqrt{1+\cos ^{2} \theta \frac{u^{2}}{h^{2}}} \mathrm{~d} \theta .
$$

Equation (6) can be solved in closed form invoking elliptic integrals [49] — a solution that still needs numerical evaluation, though. On the other hand, consistent with our stated focus on small relative meniscus perturbations $\frac{u}{h}<1$, we make use of the well-known linear approximation $(1+x)^{n} \approx 1+n x$, holding for $x \ll 1$, to replace the square root by $1+\frac{1}{2} \frac{u^{2}}{h^{2}} \cos ^{2} \theta$, finally obtaining

$$
S \approx 2 \pi R h\left(1+\frac{u^{2}}{4 h^{2}}\right) .
$$

Since the total (variable) energy of the system is here equal to

$$
E=\gamma S
$$

(where $\gamma$ is the surface tension), the lateral restoring force is equal to

$$
F=-\frac{\partial E}{\partial u}=-\pi R \gamma \frac{u}{h}
$$

which correspond to a constant lateral meniscus stiffness $K_{m}^{L}$ given by

$$
K_{m}^{L}=-\frac{\partial F}{\partial u}=\frac{\pi R \gamma}{h} .
$$

This formulation was benchmarked using our SE model, in the case of $r=50 \mu \mathrm{m}, h=70 \mu \mathrm{m}, \gamma=0.325 \mathrm{~N} \mathrm{~m}^{-1}$ and a volume of liquid given by $V=\pi r^{2} h$. The comparison is plotted in figure 7. Good agreement between the models was achieved for small relative shifts (i.e. small $u / h$ values) which are coherent with our working hypothesis and with those induced in our experiments.

\section{Results and discussion}

While performing the experiments, the magnitude of the successive bottom pad displacements $\left\{x_{d_{i}}^{b}\right\}$ was empirically determined as a compromise between two competing needs: (a) to accurately sample the curves of interest, and (b) to avoid as much as possible the effects of environmental noise. The spring was indeed sensitive enough to be clearly perturbed by the movement of the surrounding air. This was the main source of noise, together with the impulsive vibration of the translation stage (induced by floor vibrations, partly attenuated by an absorbing plastic multilayer set underneath the apparatus). As a consequence, the spring could eventually undergo stochastic swinging movements (as large as a few tens of micrometers, at maximum) before settling to the equilibrium position imposed by the boundary conditions. To cope with this, (1) we spaced the successive positions $\left\{x_{d_{i}}^{b}\right\}$ of the bottom pad up to $50 \mu \mathrm{m}$ apart; (2) we moved the 


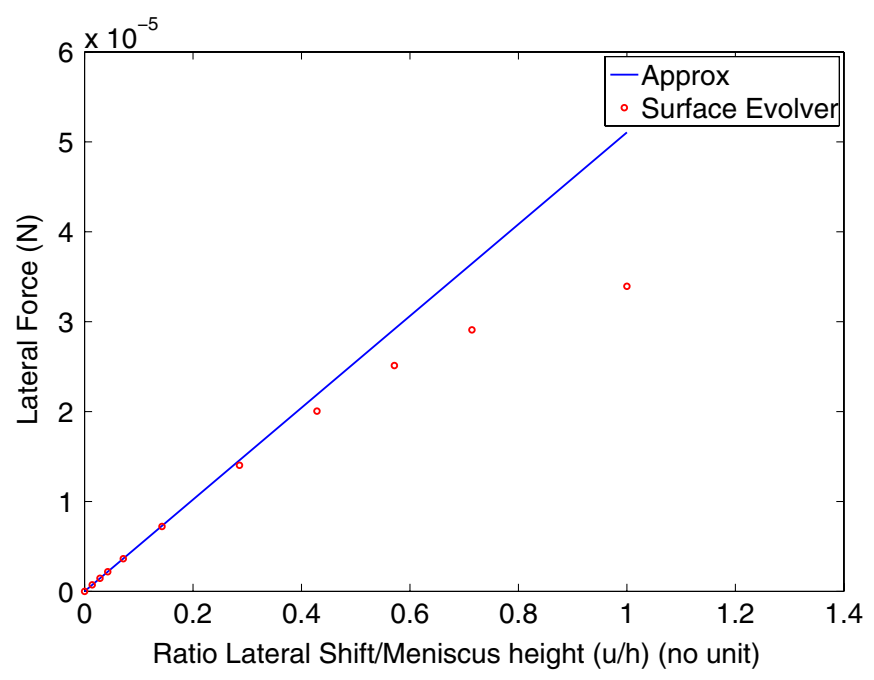

Figure 7. Comparison of analytical and numerical (SE) results for the benchmark meniscus configuration, with $R=50 \mu \mathrm{m}$, $h=70 \mu \mathrm{m}$ and $\gamma=0.325 \mathrm{~N} \mathrm{~m}^{-1}$. (Reproduced from [49]; figure 3 with kind permission from Springer Science+Business Media.)

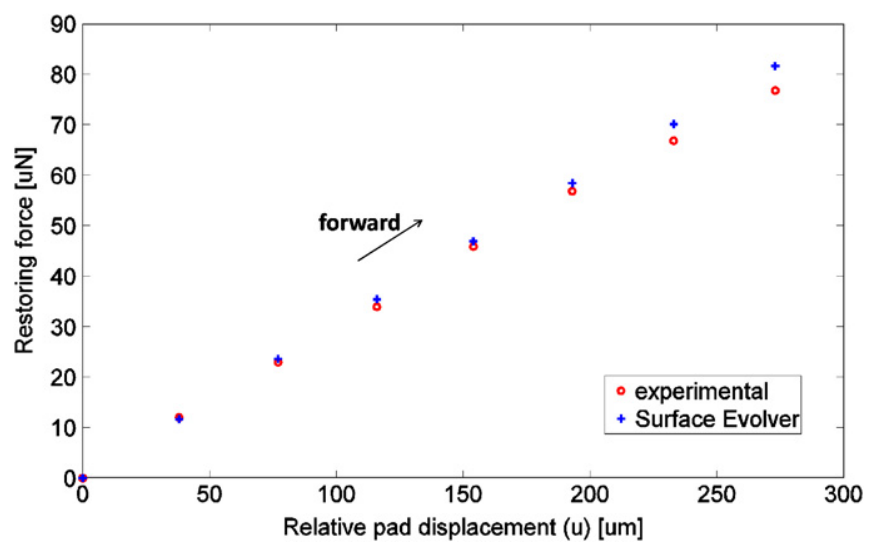

Figure 8. Experimental and numerical (SE) results for experiment 2 in table 2 .

bottom pad slowly enough in between the prefixed positions to avoid inducing excessive air and fluid flows; (3) we waited up to several tens of seconds after each prefixed position was reached to let transient air and fluid flows, and fluid velocity gradients and shear stresses inside the meniscus, go extinct: at each step, the spring settled in its new equilibrium position after undergoing a few decaying oscillations and within a time dependent on the imposed shuttle velocity and the fluid viscosity (see [49] for the detailed analysis of the system's transient dynamics); and (4) we averaged (131 072 times) the interferometric laser signals to cancel out fluctuations and thus achieve the best force resolution possible. Note that the relatively wide spacing between sampling points, as compared to the full range of imposed displacements, caused no harm to the faithful reconstruction of the desired curves: close to the origin, the behavior of the fluid menisci was indeed expected to be essentially linear $[35,36]$ — as we experimentally confirmed it to be a posteriori (see e.g. figure 8 ).

Table 2 summarizes the lateral stiffnesses of six cylindrical fluid menisci, as resulting from experiments and both

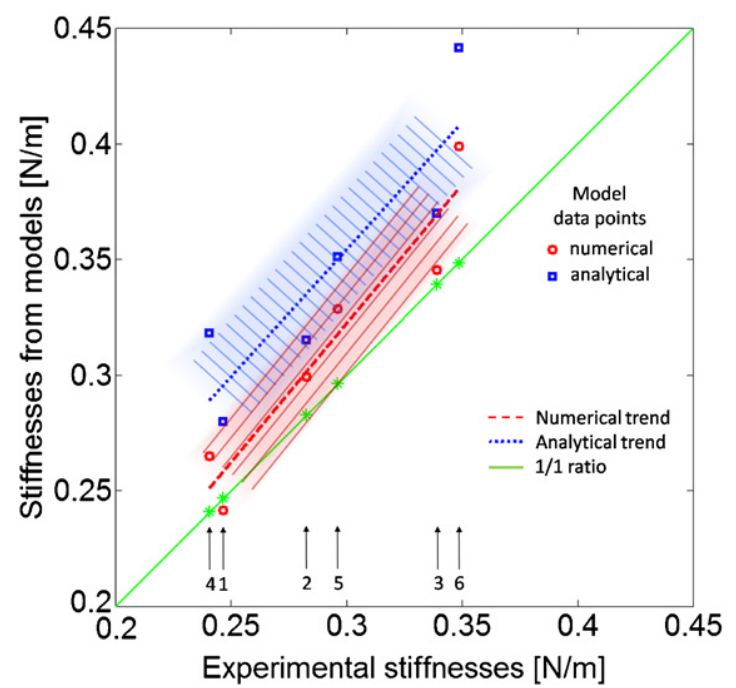

Figure 9. Comparison of lateral stiffness of six cylindrical menisci (labels refer to table 2; values ordered along abscisses), as estimated from experimental measurements and from numerical and analytical models. The trend lines represent first-order least-mean-squares fits of model data versus experimental data. It is apparent that both models tend to overestimate the meniscus stiffness.

the numerical and analytical models, together with the physical and geometrical boundary conditions and the relative estimation errors for each case. We performed a total of 34 experiments; however, we report for comparison only those experiments for which (1) no fluid overflow nor sensible evaporation was detected, and (2) the estimates of volumes and heights of the menisci were accurate (according to the criteria discussed in section 2.5). As an example, figure 8 shows both experimental and numerical data for the force-versus-lateral displacement curve relative to the second experiment reported in table 2.

The experimental results confirm that lateral capillary forces arising from cylindrical menisci of lower height (which also means lower volumes in our case, because of the adopted procedures) are proportionally larger. Furthermore, the comparison of the results reveals that both numerical and analytical models tend to overestimate the meniscus stiffness, with the numerical estimate closer to the experimental values. This is evidenced in figure 9 by the deviation of the model data trend lines from the ideal trend. The relative errors of analytical estimates (up to $32 \%$, with an average of $18.6 \%$ ) are larger than those of the numerical model (up to $14 \%$, with an average of $7.2 \%$ ), as well. We attribute these facts to several factors and sources of errors, discussed in the next section.

\subsection{Sources of error}

The relative error in the reconstructed value of pad diameters $D$ (shown in the second column of table 2) can be assumed as an indication of the error on the estimates of volumes and heights of the menisci. These errors in turn directly affect the geometry of the reconstructed menisci, and thus both models' estimates of their lateral stiffnesses. Given the relative errors 


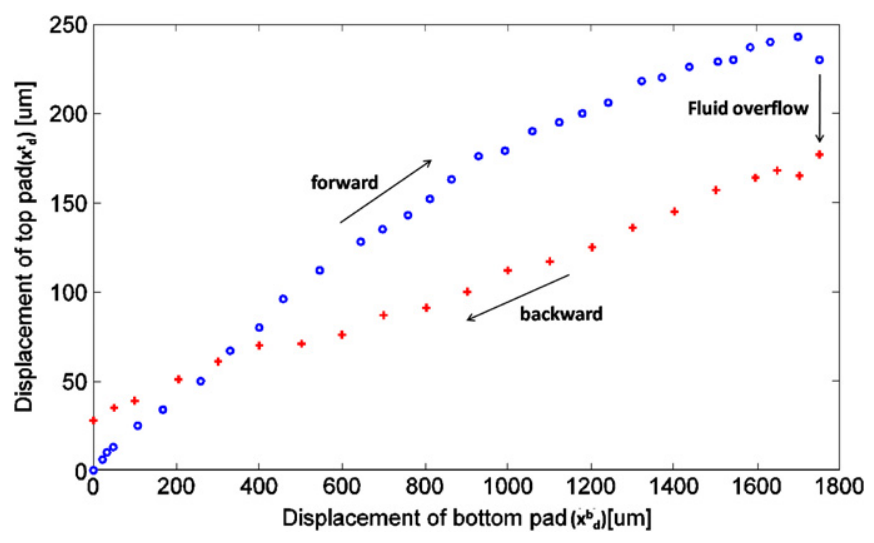

Figure 10. Experimental top pad-versus-bottom pad (i.e. $x_{d}^{t}$ versus $x_{d}^{b}$ ) displacements during forward and backward scans in the presence of hysteresis. The final large top pad displacement induced the oil overflow over the edge of the bottom pad, changing the volume and the profile of the meniscus and consequently the magnitude of the restoring force.

$\delta h / h$ and $\delta R / R$ on the estimates of $h$ and $R$, respectively, the relative error in the volume of a cylinder is

$$
\frac{\delta V}{V}=\frac{\delta h}{h}+2 \cdot \frac{\delta R}{R}
$$

which equals $3 \cdot \delta R / R$ assuming equal relative errors for $h$ and $R$. Considering e.g. the case of experiment 2 , with the reference parameter and error values shown in table 2, the effects of a $2.45 \%$ relative error in $h, D$ and $V$ give a relative error in the simulated versus experimental values of meniscus stiffness of $8.6 \%$ (for the case of errors in excess) and $7.62 \%$ (in the opposite case), respectively. This and similar error propagation analyses, as well as the little error due to mesh refinement in the model, can partly explain the relative error between SE data and real data. We also remark that the relative uncertainty in the experimental value of the stiffness of the spring ( $\sim 6 \%$, see appendix A) is by itself very close to the average relative error of the SE model compared to experiments $(7.2 \%)$.

Relative errors in estimates of $D$ do also affect, though to a lesser extent, the estimates for $K_{m}^{L}$ resulting from the analytical model. However, in this regard we could deduce a posteriori that the axisymmetric meniscus geometries we tried to enforce by visual inspection at the beginning of each experiment were not always perfectly cylindrical; we think this may be the main reason for the systematic analytical overestimates of the stiffnesses of the menisci.

Finally, a change in $\gamma$ during the experiments, as induced e.g. by adsorbtion of contaminants from air, would proportionally affect the restoring forces, thus the stiffnesses of the menisci. While this is probably the case for high surface tension liquids like water, in our contextual experiments we could not detect any significant dispersion of the surface tension of silicone oils.

In view of all these plausible sources of errors, we consider the matching between our models and experiments to be satisfactory.

\subsection{Hysteresis}

Figure 10 shows the results of one early experiment, where relatively large perturbations were imposed on an oil meniscus. Comparing forward and backward measurement curves and initial and final meniscus profiles, hysteretic behavior was clearly detected. This was due to the overflow of the fluid beyond the edge of the bottom pad (figure 11). This happened when the angle comprised between the lateral surface of the meniscus on its advancing side and the top surface of the bottom pad exceeded the limit $\zeta_{\max }$ set by Gibbs' inequality [50]:

$$
\zeta_{\max }=\theta^{a}+180^{\circ}-\phi
$$

with $\theta^{a}$ being the advancing CA of the liquid, and $\phi$ the angle between the top and the side surface of the bottom pad (approximately equal to $90^{\circ}$ in our case). Beyond this value of that angle, the fluid was no longer confined by the rim of the pad and it wet the vertical side of it, overflowing (figure 11 $(a)$ ). The overflow changed the residual amount of fluid in the meniscus. The profile of the meniscus during the backward movement was thus different compared to the forward movement, and hence there was a difference in the resulting restoring force. Depending on $\theta^{a}$ and the actual height $h$ of the meniscus, fluid overflow could be avoided by ensuring $u<u_{\max }=h \cdot \tan \left(\theta^{a}\right)$ (figure 11(b)).

We incidentally observe that, thanks to (1) the aforementioned geometrical relation between $u_{\max }, h$ and $\theta^{a}$, and (2) the possibility of exactly tracking in real time the value of the relative displacement between top and bottom pads, our setup may directly enable a method (which we call the overflow method) to experimentally determine the CA of liquids. This
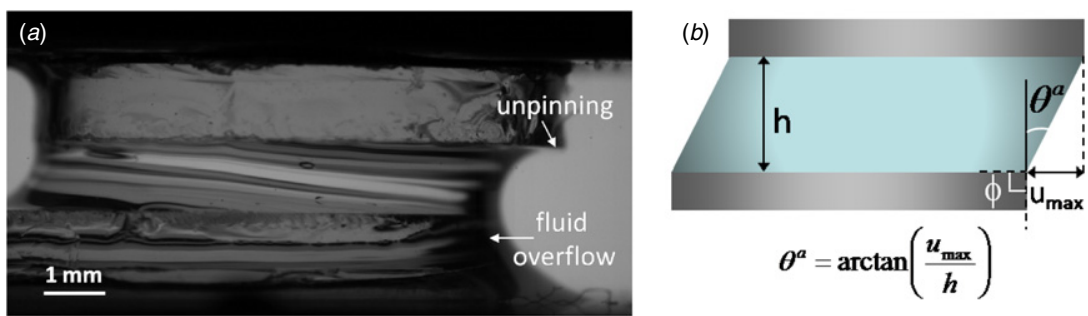

Figure 11. Hysteresis by a large displacement of the top relative to the bottom pad $(a)$. Beyond a geometrically predictable value of displacement, i.e. for an advancing angle $\theta+180-\phi$ between the fluid and the bottom pad $(b)$, the fluid is no longer confined on top of the bottom pad surface, and it overflows. The volume of the residual fluid confined between the pads decreases, changing the profile of the meniscus. Triple contact-line unpinning along the edge of the top pad is also seen. 
novel method may be suitable to measure all CAs; it may be particularly useful for estimating very small $\theta^{a}$ 's, since they are hereby 'magnified' by the additional value $180^{\circ}-\phi$ due to the constrained geometry of the setup. An actual implementation of the method would require non-circular pads with dimensions much larger than the fluid drops, to avoid stresses on the fluid, and ideal control of the solid edges of the confining surfaces. Interestingly, for this particular application no thorough calibration of our sensing apparatus would be necessary-apart from a proper synchronization of laser and visual tracking systems.

Hysteretical curves were also measured when using water as fluid, even applying only small displacements. In this case the source of hysteresis was the rapid evaporation of waterthat evidently affected the volume and thus the curvature of the meniscus - and/or the higher susceptibility of the water surface to adsoption of surfactants from air, which sensibly affect its surface tension.

\section{Conclusions}

We presented a comprehensive quasi-static study of lateral capillary forces arising from small perturbations of cylindrical fluid menisci. We experimentally measured the lateral restoring forces by means of a dedicated sensing apparatus exploiting the lateral force balance between capillary and elastic forces. We also presented an analytical model describing in closed form the lateral stiffness of a cylindrical fluid meniscus. The predictions of this model and those of a finite-element one showed a good match with the experimentally measured values of meniscus stiffnesses, the more satisfying when considering the sources of noise, error and hysteresis possibly affecting our sensing apparatus and/or intrinsic to this quasi-static type of experiments. Our results provide a solid foundation for, among others, the application of capillarity to microsystem assembly.

Our apparatus' capacity of tracking in real time and with micrometric accuracy the relative displacements of top and bottom pads may enable, given the proper boundary conditions, the direct measurement of the advancing CA of liquids. This novel method would exploit the sudden liquid overflow over the edge of the confining bottom surface induced by lateral meniscus perturbations exceeding the limit set by Gibbs' inequality. The method may be useful to assess the quality of high-energy surfaces as an alternative to interferometric methods; and robust, being based on an isolated and dramatic event. The validation of such an overflow method is currently being pursued.

Finally, our versatile experimental apparatus could constitute a reference testbed to further investigate lateral capillary forces arising from fluid menisci of arbitrary profiles and shapes. The meniscus geometry can be defined by the geometry of the confining pads-besides the other parameters discussed earlier. Scaling properties may also be studied, as well as the self-alignment dynamics [49] enabled by capillary forces. The latter is fundamental to many present-day microsystem integration technologies, from hybrid microhandling [55] to capillary part-to-substrate selfassembly [16].
Table A1. Summary of the estimates of the spring's bending stiffness $K$.

\begin{tabular}{ll}
\hline Estimation method & $K\left(\mathrm{~N} \mathrm{~m}^{-1}\right)$ \\
\hline Auxiliary cantilever-analytical & 1.1506 \\
Auxiliary cantilever-dynamic & 0.9323 \\
Auxiliary cantilever-weighting & 1.0563 \\
Dynamic & 0.9375 \\
Analytic & 0.9036 \\
\hline
\end{tabular}

\section{Acknowledgments}

This work was supported by the EU Hydromel Project. The authors thank Bruno Tartini and Jean-Salvatore Mele for their valuable help in manufacturing the mechanical components of the experimental setup.

\section{Appendix A. Bending stiffness of the spring}

We estimated the bending stiffness $K$ of our double-cantilever sliding spring in three different ways, obtaining five estimates. All geometrical parameters of the spring were known: cantilever length $L=282 \mathrm{~mm}$, width $b=12.7 \mathrm{~mm}$, thickness $t=0.102 \mathrm{~mm}$, total spring mass (including both beams, shuttle and top pad) $M=18.448 \mathrm{~g}$. Multiple alternative estimates were motivated by the uncertainty in the actual Young's modulus $E$ (standard assumed value: $210 \mathrm{GPa}$ ) and density $\rho$ (standard assumed value: $7800 \mathrm{~kg} \mathrm{~m}^{-3}$ ) of our steel cantilevers, directly affecting our analytical estimates. Good agreement between all estimates was obtained-as summarized in table A1 and detailed below. Nonetheless, we attributed the highest confidence to the two fully experimental estimates of $K$ (defined below as $K_{3}$ and $K_{4}$ ), both of which avoided the use of $E$ and $\rho$. Therefore, we assumed for the spring a bending stiffness equal to the average of $K_{3}$ and $K_{4}$, i.e. $K=0.9969 \mathrm{~N} \mathrm{~m}^{-1}$, with a relative uncertainty of $5.96 \%$.

We remark that such high sensitivity enabled both the spring's desired force resolution (see requirement (iii) of section 2.1) and its correspondingly high susceptibility to environmental perturbations (see section 5).

\section{A.1. The auxiliary cantilever method}

First estimates of $K$ involved an auxiliary steel cantilever of known dimensions (precision brand, length $l=86.4 \mathrm{~mm}$, thickness $t=0.102 \mathrm{~mm}$, width $b=12.7 \mathrm{~mm}$ ). The measurement principle exploited the lateral balance between the elastic forces of the perturbed cantilever and spring (figure A1). Starting from the initial rest position, common to both cantilever and spring, a laser-tracked lateral displacement imposed on the cantilever induced a laser-tracked lateral displacement on the spring. After determining the bending stiffness of the cantilever, the stiffness of the spring was obtained from its force-versus-displacement curve by firstorder polynomial fitting.

We estimated the bending stiffness $k$ of the auxiliary cantilever in three ways. Assuming the standard stainless 


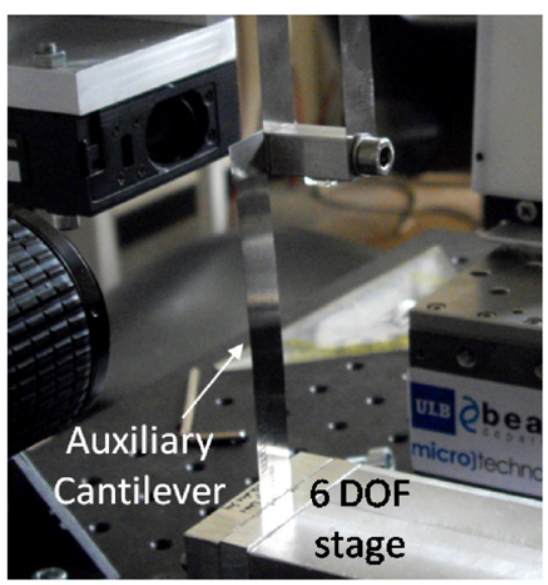

Figure A1. The auxiliary cantilever used to estimate the spring stiffness.

steel's Young's modulus $E$ and density $\rho$, the bending stiffness of a cantilever $k$ for small deformations is given analytically by (see [54])

$$
k=\frac{3 E I}{L^{3}}
$$

where $I$ is the cantilever's second moment of the area. We estimated $I$.

(i) Analytically, as $I=\frac{b t^{3}}{12}$. Inserting this into equation (A.1) leads to $k_{1}=1.097 \mathrm{~N} \mathrm{~m}^{-1}$.

(ii) From the knowledge of the first resonance $f_{1}$ of the cantilever, as obtained by solving Euler's beam equation ([54], p 273):

$$
I=\frac{2 \pi f_{1} \rho}{E \beta_{1}^{4}}
$$

where $\beta_{1}=1.875$. We measured the vibration period $t=97 \mathrm{~ms}$ of the cantilever analyzing its lasertracked oscillations on a digital oscilloscope. Hence, from equations (A.2) and (A.1) we obtained $k_{2}=$ $0.8889 \mathrm{~N} \mathrm{~m}^{-1}$.

We also estimated the bending stiffness of the cantilever by measuring and numerically fitting its tip load-versustip displacement characteristic. This gave us a value of $k_{3}=1.0071 \mathrm{~N} \mathrm{~m}^{-1}$. We considered this to be the most reliable of our estimates of $k$.

We consequently obtained one value of the bending stiffness $K_{\#}$ from each $k_{\#}$ value: $K_{1}=1.1506 \mathrm{~N} \mathrm{~m}^{-1}$, $K_{2}=0.9323 \mathrm{~N} \mathrm{~m}^{-1}$ and $K_{3}=1.0563 \mathrm{~N} \mathrm{~m}^{-1}$.

\section{A.2. The dynamic method}

From the natural oscillation frequency $f_{1}$ of the doublecantilever spring $\left(f_{1}=1.266 \mathrm{~Hz}\right.$ in our case, as measured by the oscilloscope), its stiffness $K_{4}$ can directly be estimated according to

$$
K_{4}=4 \pi^{2} f_{1}^{2} M_{\mathrm{eff}}
$$

where $M_{\text {eff }}$ is the effective spring mass, including the mass of the shuttle and the kinetic energy-averaged mass of the cantilevers (according to the Rayleigh method; see [54], p 23, and appendix B for details). We obtained $K_{4}=0.9375 \mathrm{~N} \mathrm{~m}^{-1}$.

\section{A.3. The analytical method}

We also calculated $K$ fully analytically. We assumed that $K_{5}$ had two components: (1) the mechanical stiffness of two parallel, coupled cantilevers-with their unclamped extremities constrained by the shuttle to slide along a direction perpendicular to the cantilevers-given by material strength theory [54]; and (2) a component due to the gravitational potential energy, which we converted into a socalled gravitational stiffness.

The mechanical component was obtained from

$$
K_{\text {mech }}=2 \cdot \frac{12 E I}{L^{3}} .
$$

We estimated the gravitational stiffness as (see appendix $\mathrm{C}$ for details)

$$
K_{\mathrm{grav}}=\frac{6 g}{5 L}\left(m_{s h}+\frac{m_{b}}{2}\right)
$$

where $g$ is the acceleration of gravity. All parameters being known, we got a value of $K_{5}=0.9036 \mathrm{~N} \mathrm{~m}^{-1}$.

\section{Appendix B. The effective spring mass}

The mass of the spring's two cantilevers $\left(m_{b}=5.7 \mathrm{~g}\right)$ was not negligible compared to that of the shuttle and top pad $\left(m_{s}=12.778 \mathrm{~g}\right)$. Therefore, in the dynamic estimation of the spring's stiffness we introduced an equivalent mass for both beams $m_{e q}$, which would have the same kinetic energy as the actual cantilevers for the same shuttle velocity $v$ according to

$$
\frac{1}{2} m_{e q} v^{2}=2 \cdot \frac{1}{2} \int_{0}^{L} v^{2}(z) \mathrm{d} m^{\prime}=2 \cdot \frac{1}{2} \lambda \int_{0}^{L} v^{2}(\xi) \mathrm{d} \xi
$$

where $\mathrm{d} m^{\prime}=\lambda \mathrm{d} \xi$, and $\lambda$ has the dimensions of mass per unit length. The velocity $v(z)$ of each cantilever element located at a distance $z$ from the clamped extremity was assumed to be proportional to its displacement computed by material strength theory:

$$
v(z)=\frac{q(z)}{u} v
$$

where the element $q(z)$ is given by

$$
q(z)=\frac{F}{E I}\left(\frac{L z^{2}}{4}-\frac{z^{3}}{6}\right)
$$

and $u=q(L)$. Using equations (B.3), (B.2) and (A.4) we get

$$
v^{2}(z)=v^{2}\left(\frac{9 z^{4}}{L^{4}}-\frac{12 z^{5}}{L^{5}}+\frac{4 z^{6}}{L^{6}}\right)
$$

which, inserted into equation (B.1), leads to

$$
m_{e q}=\frac{13}{35} m_{b}
$$

Finally, the effective spring mass $M_{\text {eff }}$ is given by

$$
M_{\mathrm{eff}}=m_{s h}+m_{e q}=14.8171 \mathrm{~g} \text {. }
$$




\section{Appendix C. The gravitational stiffness}

The gravitational component of the spring's stiffness arises from the fact that a horizontal displacement $u$ of the shuttle is concurrent with a vertical parasitic motion $p$ given by Henein ([56], formula 5.13) as $^{7}$

$$
p \approx \frac{3 u^{2}}{5 L}
$$

Considering that the shuttle undergoes a $p$ upward displacement while each beam's mass center undergoes a $p / 2$ vertical displacement, the gravitational stiffness $K_{\text {grav }}$ is defined as follows ( $m_{b}$ is the mass of the 2 cantilevers):

$$
\frac{1}{2} K_{\text {grav }} u^{2}=m_{s h} g p+m_{b} g \frac{p}{2}
$$

which together with equation (C.1) leads to equation (A.5).

\section{References}

[1] Adamson A W 1990 Physical Chemistry of Surfaces (New York: Wiley)

[2] de Gennes P-G, Brochard-Wyart F and Quere D 2004 Capillarity and Wetting Phenomena: Drops, Bubbles, Pearls, Waves (Berlin: Springer)

[3] Israelachvili J 1994 Intermolecular and Surface Forces (New York: Academic)

[4] Berge B and Peseux J 2000 Variable focal lens controlled by an external voltage: an application of electrowetting Eur. Phys. J. E 3 159-63

[5] De Volder M, Peirs J, Reynaerts D, Coosemans J, Puers R, Smal O and Raucent B 2005 A novel hydraulic microactuator sealed by surface tension Sensors Actuators A 123-124 547-54

[6] Lambert P 2007 Capillary Forces in Microassembly (Berlin: Springer)

[7] Wassink R J K 2005 Soldering in Electronics (Port Erin: Electrochemical Publications)

[8] Gad el Hak M (ed) 1998 The MEMS Handbook (Boca Raton, FL: CRC Press)

[9] Boncheva M and Whitesides G M 2005 Making things by self-assembly MRS Bull. 30 736-42

[10] Goodman L S 1969 Geometrical optimization of controlled collapse interconnections IBM J. Res. Dev. 13 251-65

[11] Kallmayer C, Oppermann H, Engelmann G, Zakel E and Reichl H 1996 Self-aligning flip-chip assembly using eutectic gold/tin solder in different atmospheres IEEE Int. Electronics Manufacturing Technology Symp. pp 18-25

[12] Humpston G 1997 Flip chip solder bonding for microsystems IEE Coll. on Assembly and Connections in Microsystem

[13] Tan Q and Lee Y C 1996 Soldering technology for optoelectronic packaging IEEE Electronic Components and Technology Conf. pp 26-36

[14] Syms R R A, Yeatman E M, Bright V M and Whitesides G M 2003 Surface-tension powered self-assembly of microstructures the state of the art IEEE $J$. Microelectromech. Syst. 12 387-417

[15] Guo X, Lic H, Ahn B Y, Duoss E B, Hsia K J, Lewis J A and Nuzzo R G 2009 Two- and three-dimensional folding of thin film single-crystalline silicon for photovoltaic power applications Proc. Natl Acad. Sci. USA 106 20149-54

7 Using equation (C.1), we can estimate $p$ for the top pad displacement induced by lateral force measurements. For the case of, e.g., experiment 2 of table 2, a $u_{\max }=273 \mu \mathrm{m}$, led to a negligible $p_{\max }=0.16 \mu \mathrm{m}$.
[16] Mastrangeli M, Abbasi S, Varel C, Hoof C van, Celis J-P and Bohringer K F 2009 Self-assembly from milli- to nanoscale: methods and applications J. Micromech. Microeng. 19083001

[17] Terfort A, Bowden N and Whitesides G M 1997 Three-dimensional self-assembly of millimetre-scale components Nature 386 162-4

[18] Terfort A and Whitesides G M 1998 Self-assembly of an operating electrical circuit based on shape complementarity and the hydrophobic effect Adv. Mater. 10 470-3

[19] Boncheva M, Bruzewicz D A and Whitesides G M 2003 Millimeter-scale self-assembly and its applications Pure Appl. Chem. 75 621-30

[20] Srinivasan U, Liepmann D and Howe R T 2001 Microstructure to substrate self-assembly using capillary forces IEEE $J$. Microelectromech. Syst. 10 17-24

[21] Scott K L, Hirano T, Yang H, Singh H, Howe R T and Niknejadk A N 2004 High-performance inductors using capillary based fluidic self-assembly IEEE $J$. Microelectromech. Syst. 13 300-9

[22] Xiong X, Hanein Y, Fang J, Wang Y, Wang W, Schwartz D T and Bohringer K F 2003 Controlled multibatch self-assembly of microdevices IEEE J. Microelectromech. Syst. 12 117-27

[23] Fukushima T, Yamada Y, Kikuchi H, Tanaka T and Koyanagi M 2007 Self-assembly process for chip-to-wafer three-dimensional integration IEEE Electronic Components and Technology Conf. pp 836-841

[24] Fukushima T, Kikuchi H, Yamada Y, Konno T, Liang J, Sasaki K, Inamura K, Tanaka T and Koyanagi M 2007 New three-dimensional integration technology based on rreconfigured wafer-on-wafer bonding technique IEEE Int. Electronic Device Meeting pp 985-8

[25] Stauth S A and Parviz B A 2006 Self-assembled single-crystal silicon circuits on plastic Proc. Natl Acad. Sci. USA $10313922-7$

[26] Morris C J and Parviz B A 2008 Micro-scale metal contacts for capillary force-driven self-assembly J. Micromech. Microeng. 18015022 (10pp)

[27] Zheng W and Jacobs H O 2006 Self-assembly process to integrate and interconnect semiconductor dies on surfaces with single-angular orientation and contact-pad registration Adv. Mater. 18 1387-92

[28] Chung J, Zheng W, Hatch T J and Jacobs H O 2006 Programmable reconfigurable self-assembly: parallel heterogeneous integration of chip-scale components on planar and nonplanar surfaces IEEE J. Microelectromech. Syst. 15 457-64

[29] Saeedi E, Abbasi S, Bohringer K F and Parviz B A 2006 Molten-alloy driven self-assembly for nano and micro scale system integration Fluid Dyn. Mater. Process. 2 221-45

[30] Mastrangeli M, Ruythooren W, Van Hoof C and Celis J-P 2009 Conformal dip-coating of patterned surfaces for capillary die-to-substrate self-assembly J. Micromech. Microeng. 19045015

[31] Takei A, Matsumoto K and Shimoyama I 2010 Capillary torque caused by a liquid droplet sandwiched between two plates Langmuir 26 2497-504

[32] Patra S K and Lee Y C 1991 Quasi-static modeling of the self-alignment mechanism in flip-chip soldering: Part 1. Single solder joint J. Electron. Packag. 113 337-42

[33] Patra S K and Lee Y C 1991 Modeling of self-alignment mechanism in flip-chip soldering: Part II. Multichip solder joints Proc. Electronic Components and Technology Conf. pp 783-8

[34] Yost B, McGroarty J, Borgesen P and Li C-Y 1993 Shape of a nonaxisymmetric liquid solder drop constrained by parallel plates IEEE Trans. Compon., Hybrids Manuf. Technol. 16 523-6 
[35] Lin W, Patra S K and Lee Y C 1995 Design of solder joints for self-aligned optoelectronics assembly IEEE Trans. Compon. Packag. Manuf. Technol. B 18 543-51

[36] Lieneman J, Greiner A, Korvink J G, Xiong X, Hanein Y and Bohringer K F 2003 Modelling, simulation and experiment of a promising new packaging technology parallel fluidic self-assembly of microdevices Sensors Update 13 3-43

[37] Bohringer K F, Srinivasan U and Howe R T 2001 Modelling of capillary forces and binding sites for fluidic self-assembly EEE Int. Conf. on MEMS pp 369-74

[38] Liang S-H, Xiong X and Bohringer K F 2004 Toward optimal designs for self-alignment in surface tension driven micro-assembly IEEE Int. Conf. on MEMS pp 9-12

[39] Xiong X, Liang S-H and Bohringer K F 2004 Geometric binding site design for surface-tension driven self-assembly Proc. IEEE Int. Conf. on Robotics and Automation pp 1141-8

[40] Van Veen N 1999 Analytical derivation of the self-alignment motion of flip-chip soldered components Trans. ASME 121 $116-21$

[41] Mastrangeli M 2010 Enabling capillary self-assembly for microsystem integration $P h D$ Thesis Arenberg Doctoral School, Katholieke Universiteit Leuven

[42] Sato K, Ito K, Hata S and Shimokohbe A 2003 Self-alignment of microparts using liquid surface tension: behaviour of micropart and alignment characteristics Precision Eng. 27 42-50

[43] Sato K, Lee K, Nishimura M and Okutsu K 2007 Self-alignment and bonding of microparts using adhesive droplets Int. J. Precis. Eng. Manuf. 8 75-9

[44] Bernstein R W, Zhang X, Zappe S, Fish M, Scott M and Solgaard O 2004 Characterization of fluidic microassembly for immobilization and positioning of drosophila embryos in 2-d arrays Sensors Actuators A 114 191-6
[45] Lin C, Tseng F and Chieng C-C 2008 Studies on size and lubricant effects for fluidic self-assembly of microparts on patterned substrates using capillary effect J. Electron. Packag. 130 021005-1

[46] Cooper C D and Alley F C 1994 Air Pollution Control: A Design Approach (Chicago, IL: Waveland)

[47] Zhang X, Chen C-C, Bernstein R W, Zappe S, Scott M P and Solgaard O 2005 Microoptical characterization and modeling of positioning forces on drosophila embryos self-assembled in two-dimensional arrays IEEE $J$. Microelectromech. Syst. 14 1187-97

[48] Clasen C, Gearing B P and McKinley G H 2006 The flexure-based microgap rheometer (fmr) J. Rheol. 50 883-905

[49] Lambert P, Mastrangeli M, Valsamis J-B and Degrez G 2010 Spectral analysis and experimental study of lateral capillary dynamics for flip-chip applications Microfluid. Nanofluid. DOI: $10.1007 / \mathrm{s} 10404-010-0595-2$

[50] Oliver J F, Huh C and Mason S G 1977 Resistance to spreading of liquids by sharp edges J. Colloid Interface Sci. 59 568-81

[51] Brakke K A 1992 The surface evolver Exp. Math. 1 141-65

[52] Martino P M, Freedman G M, Racz L M and Szekely J 1994 Predicting solder joint shape by computer modeling Proc. Electronic Components and Technology Conf. pp 1071-8

[53] Weatherburn C E 1955 Differential Geometry of Three Dimensions (Cambridge: Cambridge University Press)

[54] Thomson W T and Dahleh M D 1998 Theory of Vibration with Applications (Englewood Cliffs, NJ: Prentice-Hall)

[55] Sariola V, Zhou Q and Koivo H N 2008 Hybrid microhandling: a unified view of robotic handling and self-assembly J. Micro-Nano Mech. 4 5-16

[56] Henein S 2000 Conception des structures articulées à guidages flexibles de haute précision PhD Thesis Ecole Polytechnique Federale de Lousanne 